\title{
Smooth Approximation of Data on the Sphere with Splines
}

\author{
C. R. Traas, Enschede \\ Received September 29, 1986; revised December 11, 1986
}

\begin{abstract}
Zusammenfassung
Smooth Approximation of Data on the Sphere with Splines. A computable function, defined over the sphere, is constructed, which is of class $C^{1}$ at least and which approximates a given set of data. The construction is based upon tensor product spline basisfunctions, while at the poles of the spherical system of coordinates modified basisfunctions, suggested by the spherical harmonics expansion, are introduced to recover the continuity order at these points. Convergence experiments, refining the grid, are performed and results are compared with similar results available in literature.

The approximation accuracy is compared with that of the expansion in terms of spherical harmonics. The use of piecewise approximation, with locally supported basisfunctions, versus approximation with spherical harmonics is discussed.
\end{abstract}

AMS Subject Classification: 65D07.

Key words: Approximation over the sphere, tensor product splines, spherical harmonics.

Glatte Datenapproximation auf der Kugel mit Hilfe von Splines. Wir konstruieren eine berechenbare Funktion, die auf der Kugeloberfläche definiert und mindestens $C^{1}$ ist und die eine vorgegebene Datenmenge approximiert. Die Konstruktion beruht auf Tensorprodukt-Spline-Basisfunktionen; an den Polen des sphärischen Koordinatensystems benutzen wir modifizierte Basisfunktionen in Analogie zu sphärischen harmonischen Entwicklungen, um die Glattheit an diesen Stellen zu bewahren. Konvergenzexperimente mit Verfeinerung der Gitter wurden durchgeführt und die Ergebnisse mit ähnlichen in der Literatur verglichen.

Die Verwendung stückweiser Approximationen mit lokalen Basisfunktionen wird der Approximation mit sphärischen Harmonischen gegenübergestellt und die Approximationsgüten werden verglichen.

\section{Introduction}

The problem considered is to construct a computable function defined over the sphere, having continuity of class $C^{1}$ (at least) everywhere and approximating a given set of data.

Three main approaches to the solution of this problem can be distinguished when considering the literature. The first approach is the (classical) method of spherical harmonics. In fact, this method gives a function which is of smoothness class $C^{\infty}$. All elements of the underlying approximation space have global support.

A second approach is to firstly perform a mapping of the surface of the sphere to a rectangular planar region, and then to approximate in the transformed domain, 
using one of the familiar and well-established approximation techniques for a rectangular domain. This, however, gives rise to singular behaviour of the function at the poles of the sphere, an imperfection which generally is accepted.

A third approach tries, by dealing with the problem directly in the spherical setting, to avoid the polar singularities [3]. The surface of the sphere is partitioned in (triangular) elements directly, without reference to any specific direction (like a polar axis). An arbitrary, continuously differentiable function over the sphere then can be approximated in terms of its function values and its first derivatives in the vertices of the triangular grid, using a combination of several Hermite interpolants and rational weighting functions.

In the present note we concentrate on the second approach, i.e. we map the surface of the sphere onto a rectangular planar region. In the rectangular domain we choose the well-known approximation technique of tensor product splines. However, straightforward application of these splines introduces a polar singularity. We prevent the generation of this polar irregularity by introducing locally, at the poles, modified basisfunctions, the form of which is suggested by the expansion in terms of spherical harmonics. Then an overall smooth approximation is obtained, i.e. the poles are "closed". Convergence experiments, investigating the approximating surface when refining the grid, are performed and compared with similar experiments reported in the literature.

As a by-product a comparison, with respect to accuracy, is made with the expansion in terms of spherical harmonics. It turns out that, for functions which are relatively "quiet", the method of spherical harmonics gives results of the same quality at least. However, the computing effort is much higher. An important point to consider in this respect is the fill-in of the matrix of the system when approximating data. In the case of spherical harmonics this matrix is completely filled due to the global nature of the basis functions. In the case of tensor product splines the matrix is sparse due to the local nature of the basis functions. Another point which is in favour of the use of local basis functions in approximation is the fact that the coefficients associated with such basis functions are mainly determined by the local situation and, with suitable choice of basis, form a direct reflection of the shape of the curve or surface considered. It is exactly for these very reasons that one often prefers, in univariate approximation, the use of splines instead of e.g. Tchebyshev polynomials or Fourier sine and cosine functions, although the latter methods are not necessarily less accurate.

\section{The Planar Region and Functions Over the Sphere}

Let over the sphere a function $f(\theta, \phi)$ be defined, where $\theta$ and $\phi$ are the familiar spherical coordinates. We consider the following bounded region $\Omega \subset \mathbb{R}^{2}$ :

$$
\Omega=\{\theta, \phi \mid 0 \leq \theta \leq \pi, 0 \leq \phi \leq 2 \pi\} .
$$

In this region a rectangular grid is introduced, with gridpoints given by $\left(\theta_{i}, \phi_{j}\right)$, $i=0,1, \ldots, n, j=0,1, \ldots, m, \theta_{0}=\phi_{0}=0, \theta_{n}=\pi, \phi_{m}=2 \pi, \theta_{i} \leq \theta_{i+1}, \phi_{j} \leq \phi_{j+1}$, $i, j=0,1, \ldots, n-1$ and $m-1$ respectively. 
A tensor product spline approximation space is defined:

$$
S=\left\{s(\theta, \phi) \mid s=\sum_{i=-d}^{n-1} \sum_{j=-d}^{m-1} c_{i j} B_{i}(\theta) B_{j}(\phi),(\theta, \phi) \in \Omega\right\},
$$

where the functions $B_{k}($.$) are univariate polynomial B$-splines of degree $d$. The gridlines of the rectangular grid serve as knotlines for these splines. There applies:

$$
\operatorname{Supp} B_{k}(x)=\left[x_{k}, x_{k+d+1}\right] \text {. }
$$

The four boundary gridlines are taken as $(d+1)$-fold knotlines, for which the range of indexvalues is extended. We here assume that the elements $s \in S$ are of smoothness class $C^{1}$ at least.

Since our aim is to approximate data on the sphere, we must consider a subspace of $S$. In the first place we need periodicity conditions in the direction of the $\phi$-coordinate:

$$
\left.\begin{array}{l}
s(\theta, 0)=s(\theta, 2 \pi) \\
s_{\phi}(\theta, 0)=s_{\phi}(\theta, 2 \pi)
\end{array}\right\} 0 \leq \theta \leq \pi .
$$

These conditions introduce simple relations between coefficients in a subset of $\left\{c_{i j}\right\}$.

In the second place we must satisfy the pole conditions:

$$
\left.\begin{array}{l}
s(0, \phi)=C_{N}=\text { const. }(N=\text { North-pole, } \theta=0) \\
s(\pi, \phi)=C_{S}=\text { const. }(S=\text { South-pole, } \theta=\pi)
\end{array}\right\} \forall \phi \in[0,2 \pi] .
$$

This gives

$$
c_{-d, j}=C_{N}, c_{n-1, j}=C_{S}, \forall j \in[-d, m-1] .
$$

Furthermore, we require smoothness of class $C^{1}$ at the poles. Any function $f(\theta, \phi)$ which is of this smoothness class at the poles satisfies

$$
\left.\begin{array}{l}
f_{\theta}(0, \phi)=A_{N} \cos \phi+B_{N} \sin \phi \\
f_{\theta}(\pi, \phi)=A_{S} \cos \phi+B_{S} \sin \phi
\end{array}\right\}
$$

with constants $A_{N}, B_{N}, A_{S}$ and $B_{S}$.

An easy way of verifying (1) is by considering the series expansions in terms of spherical harmonics:

where

$$
f(\theta, \phi)=C_{0}(\theta)+\sum_{k=1}^{\infty}\left[C_{k}(\theta) \cos k \phi+D_{k}(\theta) \sin k \phi\right],
$$

$$
\begin{gathered}
C_{k}(\theta)=\sum_{l=k}^{\infty} a_{l k} P_{l}^{k}(\cos \theta), a_{l k} \text { constant, } k=0,1,2, \ldots \\
D_{k}(\theta)=\sum_{i=k}^{\infty} b_{l k} P_{l}^{k}(\cos \theta), b_{l k} \text { constant, } k=1,2, \ldots
\end{gathered}
$$

The functions $P_{l}^{k}(\xi)$ are the associated Legendre functions (see any textbook on spherical harmonics, e.g. [4]). 
Since

for any $l \geq k$, there applies

$$
P_{l}^{k}(1)= \begin{cases}1, & k=0 \\ 0, & k=1,2, \ldots\end{cases}
$$

and analogously

$$
f(0, \phi)=\sum_{l=0}^{\infty} a_{l 0}=\text { const. }
$$

$$
f(\pi, \phi)=\sum_{l=0}^{\infty}(-1)^{l} a_{t 0}=\text { const. }
$$

Upon differentiating (2) once with respect to $\theta$, and using one of the recursion relations valid for associated Legendre functions and their derivatives (see [4]), it is easily verified that $f_{\theta}(0, \phi)$ and $f_{\theta}(\pi, \phi)$ are of the form (1).

In fact, the formulas (1) correspond exactly with the expression for the directional derivative of a function which is continuously differentiable in the point considered.

However, a polynomial spline function $s(\theta, \phi)$ can not satisfy (1) exactly as long as not all of the coefficients $A_{N}, B_{N}, A_{S}, B_{S}$ are zero. Such a spline function, considered as a function over the sphere, then will be not differentiable at the poles.

If we impose the additional conditions

$$
\left.\begin{array}{l}
s_{\theta}(0, \phi)=-s_{\theta}(0, \phi+\pi) \\
s_{\theta}(\pi, \phi)=-s_{\theta}(\pi, \phi+\pi)
\end{array}\right\} \forall \phi \in[0, \pi],
$$

which again introduces relations between coefficients in a subset of $\left\{c_{i j}\right\}$, then $s(\theta, \phi)$ will be differentiable at the poles in the sense of Gateaux, i.e. in every direction over a pole a unique derivative exists. However, these derivatives do not define a unique tangent plane, i.e. the spline is not Frechet differentiable at the poles.

In the references [1] and [2] this situation is accepted and approximations to data on the sphere are thus made while realizing the target smoothness at the poles only approximately.

To be able to satisfy the requirement of overall Frẻchet differentiability we must locally, at the poles, abandon the idea of the piecewise polynomial nature of the approximating function. A way of doing this is as follows. The only part in $s(\theta, \phi)$ which contributes to the poles, as far as the function values and first derivatives are concerned, is the expression

$$
C_{N} B_{-3}(\theta)+\left[\sum_{j=-3}^{m-1} c_{-2, j} B_{j}(\phi)\right] B_{-2}(\theta)
$$

for the north pole $(\theta=0)$, and a similar expression for the south pole, assuming that cubic splines $(d=3)$ are used.

We require the first $\theta$-derivative of the ultimate approximating expression to have the form $A_{N} \cos \phi+B_{N} \sin \phi$ at the north pole, $A_{N}$ and $B_{N}$ being constants. Differentiating (3) once with respect to $\theta$ and substituting $\theta=0$ gives

$$
-\frac{3}{\theta_{1}} C_{N}+\left[\sum_{j=-3}^{m-1} c_{-2, j} B_{j}(\phi)\right] \frac{3}{\theta_{1}} .
$$


Replacing this expression by $A_{N} \cos \phi+B_{N} \sin \phi$, i.e. substituting

$$
C_{N}+\frac{1}{3} \theta_{1}\left(A_{N} \cos \phi+B_{N} \sin \phi\right) \text { for } \sum_{j=-3}^{m-1} c_{-2, j} B_{j}(\phi) \text { in } s(\theta, \phi),
$$

and applying the analogous process to the south pole, gives the modified spline expression:

$$
\begin{gathered}
\hat{s}(\theta, \phi)=C_{N} B_{-3}(\theta)+\left[C_{N}+\frac{\theta_{1}}{3}\left(A_{N} \cos \phi+B_{N} \sin \phi\right)\right] B_{-2}(\theta) \\
+\sum_{i=-1}^{n-3} \sum_{j=-3}^{m-1} c_{i j} B_{i}(\theta) B_{j}(\phi) \\
+\left[C_{S}-\frac{\pi-\theta_{n-1}}{3}\left(A_{S} \cos \phi+B_{S} \sin \phi\right)\right] B_{n-2}(\theta)+C_{S} B_{n-1}(\theta) .
\end{gathered}
$$

In this expression the periodicity in $\phi$-direction has not yet been included; this, however, is an elementary matter. The dimension of the underlying approximation space is $(n-1)(m+1)+6$.

Only near the poles the function $\hat{s}$ is not of piecewise polynomial nature with respect to $\phi$. Considered as a function over the sphere, $\hat{s}$ is built up everywhere of linear combinations of locally supported basis functions. In the planar region, however, $\hat{s}$ is partially of global nature with respect to $\phi$. If we approximate $\cos \phi$ and $\sin \phi$ in (4) by means of cubic splines, using the knots $\phi_{j}$ given before, then (4) reduces to an expression (consisting exclusively of local functions) which is, effectively, equivalent to the expressions used in [1] and [2]. The Frechet differentiability at the poles, however, then is lost.

The above concept can easily be extended to higher orders of smoothness. We here give in short the analysis needed for approximating from a space whose elements are of smoothness class $C^{2}$ everywhere. We again consider cubic splines. The only part in (4) which contributes to the poles, as far as the function values and the first and second derivatives are concerned, is the expression

$$
\begin{gathered}
C_{N} B_{-3}(\theta)+\left[C_{N}+\frac{1}{3} \theta_{1}\left(A_{N} \cos \phi+B_{N} \sin \phi\right)\right] B_{-2}(\theta)+ \\
+\left[\sum_{j=-3}^{m-1} c_{-1, j} B_{j}(\phi)\right] B_{-1}(\theta)
\end{gathered}
$$

for the north pole, and a similar expression for the south pole. The corresponding part in the harmonic expansion (2) is

$$
C_{0}(\theta)+\sum_{k=1}^{2}\left[C_{k}(\theta) \cos k \phi+D_{k}(\theta) \sin k \phi\right] .
$$

Differentiating (5) twice with respect to $\theta$ and substituting $\theta=0$ gives

$$
-\frac{6}{\theta_{1} \theta_{2}} C_{N}-2\left(\frac{1}{\theta_{1}}+\frac{1}{\theta_{2}}\right)\left(A_{N} \cos \phi+B_{N} \sin \phi\right)+\left[\sum_{j=-3}^{m-1} c_{-1, j} B_{j}(\phi)\right] \frac{6}{\theta_{1} \theta_{2}} .
$$


Performing the same operations with respect to (6) gives

$$
E_{N}+F_{N} \cos 2 \phi+G_{N} \sin 2 \phi
$$

with constants $E_{N}, F_{N}$ and $G_{N}$.

We require the second $\theta$-derivative of the ultimate approximating expression to have the form (8).

Replacing (7) by (8), i.e. substituting

$$
\begin{gathered}
C_{N}+\frac{1}{3}\left(\theta_{1}+\theta_{2}\right)\left(A_{N} \cos \phi+B_{N} \sin \phi\right)+\frac{1}{6} \theta_{1} \theta_{2}\left(E_{N}+F_{N} \cos 2 \phi+G_{N} \sin 2 \phi\right) \\
\text { for } \sum_{j=-3}^{m-1} c_{-1, j} B_{j}(\phi) \text { in (4), }
\end{gathered}
$$

and applying the analogous process to the south pole, gives the desired modified spline expression, which is such that the approximation is of smoothness class $C^{2}$ everywhere, even at the poles. The dimension of the underlying approximation space is $m(n-3)+12$.

\section{Some Numerical Experiments}

Our aim with respect to the numerical experiments in the first place was to determine the rate of convergence of the approximation to the original function (which generates the data on the sphere) when refining the grid. This is motivated by convergence results published in [2]. Our first test case is one of the surfaces of revolution used in [2]:

$$
\rho=\frac{\cos z}{\sqrt{\frac{\pi^{2}}{4}-z^{2}}}, z \in\left[-\frac{\pi}{2}, \frac{\pi}{2}\right]
$$

where $\rho$ is the cylindrical radius and $z$ is the axial coordinate. This surface has been approximated in dependence of the spherical angles $\theta$ and $\phi$, using the $C^{1}$-class representation (4) and equidistant grids. The grid fineness ranged from $(n, m)=(8,12)$ to $(12,18)$, corresponding with dimensions ranging from 97 to 215 . The approximation was in the sense of least squares, with the number of data exceeding the dimension with a factor of 5 at least. To determine in a reliable way the rate of convergence, in terms of a power of a linear dimension $h$ which is characteristic of the grid fineness, the ratio $h_{1} / h_{2}$ of the characteristic meshwidths of two grids should be not too close to 1 , otherwise the result will be very sensitive to errors in the evaluation of error norms. Due to limitations in computer capacity we were restricted to a maximum value of 1.5 for this ratio. With a very careful evaluation of the error norms of the results obtained with these extreme meshes, we arrive at the following table

\begin{tabular}{c|c} 
grid & $\|$ error $\|_{\infty}$ \\
\hline $8 \times 12$ & 0.032 \\
$12 \times 18$ & 0.0065
\end{tabular}


The largest approximation errors appeared to occur at the poles. From the table it turns out that the approximation error behaves like $O\left(h^{4}\right)$, as can be expected for bicubic least squares approximation of sufficiently smooth functions. This, however, is in contrast to the experience in [2], where a convergence rate $O\left(h^{3}\right)$ is reported. On closer investigation, in [2], it appears that the lower order convergence rate occurs at the poles, while in the equatorial region the rate is $O\left(h^{4}\right)$.

Our second test case is a potential function generated by two point masses, one point of unit mass placed in the origin of the system of coordinates, and a second point of mass 0.2 fixed at a distance 0.1. Again the representation (4) has been used, to approximate the potential function over a spherical surface of radius 0.2 . The error norms are given in the following table:

\begin{tabular}{c|c} 
grid & $\|$ error $\|_{\infty}$ \\
\hline $8 \times 12$ & 0.016 \\
$12 \times 18$ & 0.0033
\end{tabular}

which corresponds with an $O\left(h^{4}\right)$ behavior. The magnitude of the potential over this spherical surface ranges from 5.7 to 7.0 .

The above results strongly suggest that the better treatment at the poles, as performed in this report, are a reason for the obtained higher order convergence rate.

In addition, a few experiments have been done with the expansion in terms of spherical harmonics, applied to the problem of approximating a potential function. Taking care that the dimensions of both approximation spaces are almost equal, it turns out that for the radius $r=0.2$ (see the above test case) comparable accuracies are obtained. However, increasing the radius of the test sphere, soon a situation is attained in which the expansion in spherical harmonics appears to be much more accurate. This is not surprising, may be, since both a potential function and the spherical harmonics are solutions to Laplace's equation. But also for other (nonpotential) functions the expansion behaves well, as far as accuracy is concerned. The computing effort for evaluating the approximation, however, is high in comparison with that of the splines method. We found factors of up to 20 , in this respect, when using approximation spaces of dimension around 100 . The same phenomenon is reported in [1].

A conclusion from these additional experiments therefore is that the choice of the splines method cannot be motivated, in general, on the basis of accuracy but, rather, on the basis of computing economy and of the favourable properties of locally supported approximation functions, as mentioned in the introduction.

\section{References}

(1) Dierckx, P.: Algorithms for smootbing data on the sphere with tensor product splines. Computing $32,319-342$ (1984).

[2] Gmelig Meyling, R. H. J., Pfluger, P. R.: B-spline approximation of a closed surface. Report 84-16, University of Amsterdam, 1984. To appear in IMA J. of Num. Analysis. 
[3] Lawson, C. L.: $C^{1}$ surface interpolation for scattered data on a sphere. Rocky Mountain J. of Math. 14, 177-202 (1984).

[4] MacRobert, T. M.: Spherical Harmonics. Pergamon Press, 1967.

Dr. C. R. Traas

University of Twente

Faculty of Applied Mathematics

P.O. Box 217

NL-7500 AE Enschede

The Netherlands

Verleger: Springer-Verlag KG, Mölkerbastei 5, A-1010 Wien. - Herausgeber: Prof. Dr. Hans J. Stetter, Institut für Angewandte und Numerische Mathematik der Technischen Universität Wien, Wiedner Hauptstraße 6-10, A-1040 Wien. - Redaktion: Wiedner Hauptstraße 6-10, A-1040 Wien. - Hersteller: Satz Austro-Filmsatz Richard Gerin, Zirkusgasse 13, A-1020 Wien, Druck Paul Gerin, Zirkusgasse 13, A-1021 Wien. - Verlagsort: Wien. Herstellungsort: Wien. - Printed in Austria. 